Check for updates

Cite this: RSC Adv., 2017, 7, 19136

Received 14th February 2017

Accepted 17th March 2017

DOI: $10.1039 / c 7 r a 01850 a$

rsc.li/rsc-advances

\section{Effect of fluoro-polycarbonates containing aliphatic/aromatic segments on the characteristics of thermo-optic waveguide devices $\dagger$}

\author{
Zhenzhen Cai, ${ }^{a}$ Qixuan Yu, ${ }^{a}$ Yang Zheng ${ }^{\mathrm{C}}$ Xiaoyu Shi, ${ }^{a}$ Xuesong Wang, ${ }^{a}$ \\ Zhanchen Cui, $\mathbb{D}{ }^{* a b}$ Zuosen Shi, ${ }^{* a}$ Changming Chen $^{c}$ and Daming Zhang ${ }^{c}$
}

Fluorinated polycarbonates containing dodecyl groups (AF-Ali-PC EPs), biphenyl groups (AF-Ar-PC EPs), and cross-linkable epoxy groups were synthesized to investigate the effects of the flexibility of monomers on the properties of thermo-optic waveguide devices. Transparent polymer films were prepared via photocuring of a solution (FPRs) containing AF-Ali-PC EPs or AF-Ar-PC EPs, a photo initiator, and cyclopentanone. The optical films have low surface energies and roughnesses, high thermal stabilities, and tunable refractive indices. Straight optical waveguide and MZI thermo-optic waveguide switch arrays were fabricated using Ali-PR 1 and Ar-PR 1. The propagation losses of the channel waveguides, measured by the cutback method, were $0.21 \mathrm{~dB} \mathrm{~cm}$ cm $^{-1}$ and $0.19 \mathrm{~dB} \mathrm{~cm}^{-1}$ at the monitoring light of $1550 \mathrm{~nm}$ for the Ali-PR 1 and Ar-PR 1, respectively. The rise and fall times of the thermo-optic switch upon application of a $200 \mathrm{~Hz}$ square-wave voltage were $1.822 \mathrm{~ms}$ and $1.364 \mathrm{~ms}$, respectively, for Ali-PRs and $2.994 \mathrm{~ms}$ and $2.301 \mathrm{~ms}$, respectively, for Ar-PRs at the square-wave voltage of $110 \mathrm{~Hz}$. The insertion loss and extinction ratio were measured to be $9.2 \mathrm{~dB}$ and $12.0 \mathrm{~dB}$ for the aliphatic segmentcontaining photoresists and $8.9 \mathrm{~dB}$ and $12.3 \mathrm{~dB}$ for the aromatic segment-containing photoresists, respectively. The applied electric powers were $15.0 \mathrm{~mW}$ and $20.0 \mathrm{~mW}$, which were also the switching powers. In contrast with our previous work, fluoro-polycarbonates containing aliphatic segments were more suitable for optical waveguide devices than those containing aromatic segments.

\section{Introduction}

Optical switches, which are implemented to establish reconfigurable connections between multiple sources and multiple destinations, are essential building blocks for both long-haul optical communications and short-reach photonic networkson-chips. ${ }^{1}$ Nowadays, optical switches and their related arrays are widely used in optical cross connects (OXCs), optical adddrop multiplexers (OADMs), optical network monitoring, and on-chip optical interconnections. ${ }^{2}$ It is desirable for these switches to have large optical bandwidths, small physical dimensions, and good performance. ${ }^{3}$ To realize these properties, various device structures such as Mach-Zehnder interferometer (MZI) switches, multimode interference (MMI) switches, and directional coupler (DC) switches have been employed. ${ }^{4}$

${ }^{a}$ State Key Laboratory of Supramolecular Structure and Materials, Jilin University, Changchun 130012, People's Republic of China. E-mail: cuizc@jlu.edu.cn; shizs@ jlu.edu.cn

${ }^{b}$ Department of Chemistry and Pharmacy, Zhuhai College of Jilin University, Zhuhai 519041, People's Republic of China

'State Key Lab on Integrated Opto-electronics, Jilin University Region, Changchun 130012, People's Republic of China

$\dagger$ Electronic supplementary information (ESI) available. See DOI: $10.1039 / \mathrm{c} 7 \mathrm{ra} 01850 \mathrm{a}$
The thermo-optic MZI is a basic device in planar light-wave circuits. In optical communication systems, it is widely used in optical modulators or switches because of its low optical loss, polarization independence, and long-term stability. ${ }^{5}$ Polymer materials have been intensively investigated due to their potential applications in optical waveguides because of their excellent characteristics ${ }^{4}$ such as low optical loss, high extinction ratio, small cubage, easy integration, high thermal and environmental stability, and flexible refractive index controllability. ${ }^{6}$ Various organic polymers including polyimides, acrylic polymers, polycarbonates, polystyrenes, polysiloxanes, hyperbranched polymers, perfluorinated methacrylates, and bis-phenol-A novolac resin (SU-8) have been tested as optical waveguide materials. ${ }^{7}$

In general, glass and silicon wafers are selected as substrates for the fabrication of optical waveguides. However, $\mathrm{Si}-\mathrm{O}$ or $\mathrm{Si}-$ $\mathrm{OH}$ groups on the surfaces of the substrates result in highly polar surfaces; moreover, it is difficult to obtain good adhesion with polymers. To solve this problem, polar functional groups, such as carbonate groups, can be introduced to enhance the adhesion of the polymer layer to the substrates. ${ }^{7 g}$ Apart from this, the properties such as transparency, broad temperature resistance, inherent toughness, and high index of refraction of polycarbonates have increased their popularity in the optical waveguide field. ${ }^{8}$ However, polycarbonates have relatively high 
absorption losses at optical communication wavelengths $(0.6 \mathrm{~dB}$ $\mathrm{cm}^{-1}$ at $1550 \mathrm{~nm}$ ), resulting from the absorption of C-H bonds. ${ }^{7 f}$ Replacing $\mathrm{C}-\mathrm{H}$ bonds with $\mathrm{C}-\mathrm{F}$ bonds in polymers can reduce the intrinsic optical loss at the key telecommunication wavelengths of 1310 and $1550 \mathrm{~nm} .{ }^{10}$ Fluorine-containing polymers exhibit good thermal and chemical stability and decreased intermolecular attractive forces (which results in better solubility) compared to hydrocarbon polymers. ${ }^{7 g, 10 a, 11}$ The refractive indices of the polymers that we have synthesized ${ }^{9}$ (AF-Z-PC EPs) can be adjusted by controlling the content of bisphenol Z. For AF-Z-PC EPs containing epoxy groups, we utilized a simple method to fabricate the waveguide devices. Encouraged by these results, we designed another experiment for further exploration.

The crystallinity of a polymer depends on its chain flexibility. Bisphenol-A polycarbonate (BAPC) presents poor crystallization because of its rigidity. ${ }^{12}$ In this study, a series of fluoropolycarbonates containing aliphatic segments (AF-Ali-PC EPs) and biphenyl segments (AF-Ar-PC EPs) were synthesized with the intention of investigating the effects of the flexibility of the monomers on the characteristics of thermo-optic waveguide devices. AF-Ali-PC EPs and AF-Ar-PC EPs were mixed with cyclopentanone and a photoinitiator, respectively, to prepare a series of negative-type fluorinated photoresists (Ali-PRs and Ar-PRs, respectively). Through direct UV-writing, the photoresists could be fabricated into straight optical waveguide and MZI thermo-optic waveguide switch arrays. The propagation losses of the channel waveguides, measured by the cutback method, were $0.21 \mathrm{~dB} \mathrm{~cm}^{-1}$ and $0.19 \mathrm{~dB} \mathrm{~cm}^{-1}$ at the monitoring light of $1550 \mathrm{~nm}$ for the Ali-PR 1 and Ar-PR 1, respectively. The rise and fall times of the thermo-optic switch upon application of a $200 \mathrm{~Hz}$ square-wave voltage were $1.822 \mathrm{~ms}$ and $1.364 \mathrm{~ms}$, respectively, for Ali-PRs and $2.994 \mathrm{~ms}$ and $2.301 \mathrm{~ms}$, respectively, for Ar-PRs at the square-wave voltage of $110 \mathrm{~Hz}$. The insertion loss and extinction ratio were measured to be $9.2 \mathrm{~dB}$ and $12.0 \mathrm{~dB}$, respectively, for the aliphatic segment-containing photoresists and $8.9 \mathrm{~dB}$ and $12.3 \mathrm{~dB}$, respectively, for the aromatic segment-containing photoresists. The applied electric power was $15.0 \mathrm{~mW}$ and $20.0 \mathrm{~mW}$.

\section{Experimental}

\section{Materials}

4,4'-(Hexafluoroisopropylidene) diphenol (98\%), 4,4'-dihydroxydiphenyl (97\%), and triphosgene (99\%) were purchased from Aladdin Industrial Co. and used as received. Dodecanedioic acid (99\%) (Sigma-Aldrich), thionyl chloride (99.5\%) (Fuchen Chemical Co., Ltd), and triphenylsulfonium hexafluorophosphate (Sigma-Aldrich) were used as received without further purification. Epoxy chloropropane, pyridine, and dichloromethane were purchased from Beijing Chemical Co., Ltd and were used after purification.

\section{Measurements}

IR spectrwerea were obtained via an AVATAR 360 spectrophotometer using $\mathrm{KBr}$ pellets. UV-vis-NIR spectra were acquired using a SHIMADZU UV-3600 spectrometer. ${ }^{1} \mathrm{H}$ NMR, ${ }^{13} \mathrm{C}$ NMR, and ${ }^{19} \mathrm{~F}$ NMR spectra were obtained via a Bruker AVANCE NMR spectrometer, operated at 500,125 , or $300 \mathrm{MHz}$, respectively. ${ }^{1} \mathrm{H},{ }^{13} \mathrm{C}$, and ${ }^{19} \mathrm{~F}$ chemical shifts have been reported as values (ppm) relative to $\mathrm{CDCl}_{3}$ (7.24). The molecular weights of the polymers were determined relative to polystyrene standards using gel permeation chromatography (GPC) in THF at room temperature. Thermal analyses were conducted via a NETZSCH 4 differential scanning calorimeter (DSC) and a Perkin-Elmer TGA 7 thermogravimetric analyzer (TGA) at the heating rate of $10{ }^{\circ} \mathrm{C} \mathrm{min}^{-1}$ under a nitrogen atmosphere. Cross-section images of the specimens were obtained via a scanning electron microscope (JEOL FESEM 6700F). An M-2000VI ellipsometer was used to determine the refractive indices of the films. The surface morphologies of the films were investigated under ambient conditions using a Digital Instruments Nanoscope IIIa instrument.

\section{Synthesis of the polymers}

Synthesis of hydroxyl-terminated fluoro-polycarbonates containing aliphatic segments. The preparation procedure has been described in our previous work., ${ }^{9,13}$ The typical procedure is as follows: a round-bottomed flask was charged with dodecanedioic acid (DDDA) $(0.96 \mathrm{~g}, 3.0 \mathrm{mmol})$ and thionyl chloride (1 $\mathrm{mL}, 14 \mathrm{mmol}$ ). The mixture was heated to the reflux temperature, and the temperature was maintained for $4 \mathrm{~h}$. After the reaction completed, the organic phase was removed under reduced pressure, and dodecanedioyl dichloride was obtained. 4,4'-(Hexafluoroisopropylidene) diphenol (6FBPA) (2.69 g, 8.0 $\mathrm{mmol}$ ) was dissolved in $0.48 \mathrm{~mL}$ of pyridine and methylene chloride and then cooled down to $0{ }^{\circ} \mathrm{C}$; after this, dodecanedioyl dichloride was added dropwise. The reaction mixture was vigorously stirred at $0-5{ }^{\circ} \mathrm{C}$ for $10 \mathrm{~min}$ and at room temperature for $2 \mathrm{~h}$. Finally, 6FBPA, pyridine, and methylene chloride were added to the reaction flask. The solution was cooled down to $0{ }^{\circ} \mathrm{C}$. A solution of triphosgene in methylene chloride was then added dropwise, and the reaction mixture was stirred for another $6 \mathrm{~h}$ at room temperature. After this, the solution was precipitated in methanol $(400 \mathrm{~mL})$, and the precipitates were obtained by filtration. The products were dried under vacuum and were denoted as AF-Ali-PC OHs.

AF-Ali-PC OH 1: 6FBPA (13.44 g, $40 \mathrm{mmol})$, pyridine $(6.0 \mathrm{~mL})$, and triphosgene $(3.71 \mathrm{~g}, 12.5 \mathrm{mmol})$. AF-Ali-PC OH 1 was obtained as a white powder $(11.59 \mathrm{~g}, 65 \%)$. GPC: $M_{\mathrm{n}}=11892 \mathrm{~g}$ $\mathrm{mol}^{-1} ; M_{\mathrm{w}} / M_{\mathrm{n}}=1.20$. IR $\left(\mathrm{KBr}, \mathrm{cm}^{-1}\right): \gamma(\mathrm{C}-\mathrm{F})=1177-1239$, $\gamma($ aromatic $)=1607$ and 1509, $\gamma(\mathrm{CO}-\mathrm{O}-\mathrm{Ar})=1777, \gamma\left(-\mathrm{CH}_{2}-\right)=$ 1712 and 732. ${ }^{1} \mathrm{H}$ NMR (500 MHz, $\left.\mathrm{CDCl}_{3}\right): \delta(\mathrm{ppm}): 7.51-7.50(\mathrm{t}$, $11 \mathrm{H}, \mathrm{ArH}), 7.44-7.43(\mathrm{t}, 1 \mathrm{H}, \mathrm{ArH}), 7.37-7.36(\mathrm{t}, 11 \mathrm{H}, \mathrm{ArH}), 7.16-$ $7.13(\mathrm{t}, 1 \mathrm{H}, \mathrm{ArH}), 2.62-2.59\left(\mathrm{~m}, 1 \mathrm{H},-\mathrm{CH}_{2}\right), 1.82-1.75(\mathrm{~m}, 1 \mathrm{H}$, $\left.-\mathrm{CH}_{2}\right), 1.44-1.36\left(\mathrm{~m}, 3 \mathrm{H},-\mathrm{CH}_{2}\right) \cdot{ }^{13} \mathrm{C}$ NMR $\left(125 \mathrm{MHz}, \mathrm{CDCl}_{3}\right)$, $\delta$ (ppm): 171.93, 151.22, 151.17, 131.69, 131.28, 123.94, 121.53, $120.77,120.52,115.16,64.00,34.37,29.39,29.23,29.07,24.86$. $\left.{ }^{19} \mathrm{~F} \mathrm{NMR} \mathrm{(470} \mathrm{MHz,} \mathrm{CDCl}_{3}, \mathrm{CFCl}_{3}\right), \delta(\mathrm{ppm}):-63.87\left(6 \mathrm{~F},-\mathrm{CF}_{3}\right)$.

AF-Ali-PC OH 2: 6FBPA $(7.392 \mathrm{~g}, 22 \mathrm{mmol})$, pyridine $(3.6 \mathrm{~mL})$, and triphosgene $(2.227 \mathrm{~g}, 7.5 \mathrm{mmol})$. AF-Ali-PC OH 2 was obtained as a white powder $(7.94 \mathrm{~g}, 68 \%)$. GPC: $M_{\mathrm{n}}=11541 \mathrm{~g}$ $\mathrm{mol}^{-1} ; M_{\mathrm{w}} / M_{\mathrm{n}}=1.31$. IR $\left(\mathrm{KBr}, \mathrm{cm}^{-1}\right): \gamma(\mathrm{C}-\mathrm{F})=1174-1242$, 
$\gamma($ aromatic $)=1605$ and 1508, $\gamma(\mathrm{CO}-\mathrm{O}-\mathrm{Ar})=1777, \gamma\left(-\mathrm{CH}_{2}-\right)=$ 1709 and 736. ${ }^{1} \mathrm{H}$ NMR (500 MHz, $\left.\mathrm{CDCl}_{3}\right), \delta(\mathrm{ppm}): 7.51-7.50(\mathrm{t}$, $8 \mathrm{H}, \mathrm{ArH}), 7.44-7.43(\mathrm{t}, 1 \mathrm{H}, \mathrm{ArH}), 7.37-7.35$ (t, 8H, ArH), 7.16$7.14(\mathrm{t}, 1 \mathrm{H}, \mathrm{ArH}), 2.62-2.59\left(\mathrm{~m}, 1 \mathrm{H},-\mathrm{CH}_{2}\right), 1.81-1.75(\mathrm{~m}, 1 \mathrm{H}$, $\left.-\mathrm{CH}_{2}\right), 1.44-1.36\left(\mathrm{~m}, 3 \mathrm{H},-\mathrm{CH}_{2}\right) .{ }^{13} \mathrm{C} \mathrm{NMR}\left(125 \mathrm{MHz}, \mathrm{CDCl}_{3}\right)$, $\delta$ (ppm): 172.01, 151.22, 131.69, 131.39, 131.28, 122.17, 121.48, 120.77, 120.52, 115.18, 64.00, 34.37, 29.39, 29.23, 29.07, 24.86. $\left.{ }^{19} \mathrm{~F} \mathrm{NMR} \mathrm{(470} \mathrm{MHz,} \mathrm{CDCl}_{3}, \mathrm{CFCl}_{3}\right), \delta(\mathrm{ppm}):-63.87\left(6 \mathrm{~F},-\mathrm{CF}_{3}\right)$.

AF-Ali-PC OH 3: 6FBPA (3.36 g, $10 \mathrm{mmol})$, pyridine $(2.0 \mathrm{~mL})$, triphosgene $(1.238 \mathrm{~g}, 4.17 \mathrm{mmol})$. AF-Ali-PC OH 3 was obtained as a white powder $(5.04 \mathrm{~g}, 66 \%)$. GPC: $M_{\mathrm{n}}=11568 \mathrm{~g} \mathrm{~mol}^{-1} ; M_{\mathrm{w}} /$ $M_{\mathrm{n}}=1.35$. IR $\left(\mathrm{KBr}, \mathrm{cm}^{-1}\right): \gamma(\mathrm{C}-\mathrm{F})=1163-1239, \gamma($ aromatic $)=$ 1612 and 1515, $\gamma(\mathrm{CO}-\mathrm{O}-\mathrm{Ar})=1784, \gamma\left(-\mathrm{CH}_{2}\right)=1715$ and $736 .{ }^{1} \mathrm{H}$ NMR (500 MHz, $\mathrm{CDCl}_{3}$ ), $\delta(\mathrm{ppm}): 7.51-7.50$ (t, 5H, ArH), 7.447.43 (t, 1H, ArH), 7.37-7.36 (t, 5H, ArH), 7.16-7.14 (t, 1H, ArH), 2.62-2.59 (m, $\left.1 \mathrm{H},-\mathrm{CH}_{2}\right), 1.81-1.75\left(\mathrm{~m}, 1 \mathrm{H},-\mathrm{CH}_{2}\right), 1.44-1.36(\mathrm{~m}$, $\left.3 \mathrm{H},-\mathrm{CH}_{2}\right) .{ }^{13} \mathrm{C}$ NMR $\left(125 \mathrm{MHz}, \mathrm{CDCl}_{3}\right), \delta(\mathrm{ppm}): 171.94,151.22$, 151.16, 131.70, 131.40, 130.33, 123.95, 121.54, 120.78, 115.15, 64.00, 34.37, 29.39, 29.24, 29.08, 24.86. ${ }^{19} \mathrm{~F}$ NMR (470 MHz, $\left.\mathrm{CDCl}_{3}, \mathrm{CFCl}_{3}\right), \delta(\mathrm{ppm}):-63.87\left(6 \mathrm{~F},-\mathrm{CF}_{3}\right)$.

Synthesis of hydroxyl-terminated fluoro-polycarbonates containing aromatic segments. To a $250 \mathrm{~mL}$ three-neck flask, 6FBPA, 4,4'-dihydroxydiphenyl (DOD, $6 \mathrm{mmol}, 1.116 \mathrm{~g}$ ), pyridine, and tetrahydrofuran were added. The solution was cooled down to $0{ }^{\circ} \mathrm{C}$. A solution of triphosgene in tetrahydrofuran was added dropwise, and the reaction mixture was stirred for another $6 \mathrm{~h}$ at room temperature. After this, the solution was precipitated in methanol $(400 \mathrm{~mL})$, and the precipitates were obtained by filtration. The products were dried under vacuum and were denoted as AF-Ar-PC OHs.

AF-Ar-PC OH 1: 6FBPA (16.13 g, $48 \mathrm{mmol})$, pyridine $(7.3 \mathrm{~mL})$, and triphosgene $(4.46 \mathrm{~g}, 15 \mathrm{mmol})$. AF-Ar-PC OH 1 was obtained as a white powder $(11.22 \mathrm{~g}, 61 \%)$. GPC: $M_{\mathrm{n}}=9894 \mathrm{~g} \mathrm{~mol}^{-1} ; M_{\mathrm{w}} /$ $M_{\mathrm{n}}=1.32$. IR $\left(\mathrm{KBr}, \mathrm{cm}^{-1}\right): \gamma(\mathrm{C}-\mathrm{F})=1173-1211, \gamma($ aromatic $)=$ 1609 and 1512, $\gamma(\mathrm{CO}-\mathrm{O}-\mathrm{Ar})=1782 .{ }^{1} \mathrm{H} \mathrm{NMR}\left(500 \mathrm{MHz}, \mathrm{CDCl}_{3}\right)$ : $\delta(\mathrm{ppm}): 7.66-7.64(\mathrm{t}, 3 \mathrm{H}, \mathrm{ArH}), 7.51-7.49(\mathrm{t}, 17 \mathrm{H}, \mathrm{ArH}), 7.40-$ 7.35 (m, 20H, ArH), 7.27 (s, 1H, ArH), 6.82 (s, 1H, ArH). ${ }^{13} \mathrm{C}$ NMR (125 MHz, $\mathrm{CDCl}_{3}$ ), $\delta$ (ppm): 156.20, 151.33, 151.22, 150.44, 138.50, 131.69, 131.27, 128.38, 123.95, 121.26, 120.76, 120.52, 115.17, 64.01. ${ }^{19} \mathrm{~F} \mathrm{NMR}\left(470 \mathrm{MHz}, \mathrm{CDCl}_{3}, \mathrm{CFCl}_{3}\right), \delta$ (ppm): $-63.86\left(6 \mathrm{~F},-\mathrm{CF}_{3}\right)$.

AF-Ar-PC OH 2: 6FBPA (12.10 g, $36 \mathrm{mmol})$, pyridine (5.65 $\mathrm{mL}$ ), and triphosgene $(3.47 \mathrm{~g}, 11.7 \mathrm{mmol})$. AF-Ar-PC OH 2 was obtained as a white powder $(8.33 \mathrm{~g}, 59 \%)$. GPC: $M_{\mathrm{n}}=8057 \mathrm{~g}$ $\mathrm{mol}^{-1} ; M_{\mathrm{w}} / M_{\mathrm{n}}=1.29$. IR $\left(\mathrm{KBr}, \mathrm{cm}^{-1}\right): \gamma(\mathrm{C}-\mathrm{F})=1176-1215$, $\gamma($ aromatic $)=1612$ and 1512, $\gamma(\mathrm{CO}-\mathrm{O}-\mathrm{Ar})=1776 .{ }^{1} \mathrm{H}$ NMR $(500$ $\left.\mathrm{MHz}, \mathrm{CDCl}_{3}\right): \delta(\mathrm{ppm}): 7.66-7.64(\mathrm{t}, 3 \mathrm{H}, \mathrm{ArH}), 7.51-7.50(\mathrm{t}, 12 \mathrm{H}$, ArH), 7.40-7.36 (m, 15H, ArH), 7.27 (s, 1H, ArH), 6.81 (s, 1H, ArH). $\left.{ }^{13} \mathrm{C} \mathrm{NMR} \mathrm{(125} \mathrm{MHz,} \mathrm{CDCl}_{3}\right), \delta$ (ppm): 156.25, 151.34, 151.22, 150.46, 138.50, 131.69, 131.29, 128.38, 123.94, 121.33, 121.26, 120.76, 115.17, 63.97. ${ }^{19} \mathrm{~F}$ NMR (470 $\mathrm{MHz}, \mathrm{CDCl}_{3}$, $\left.\mathrm{CFCl}_{3}\right), \delta(\mathrm{ppm}):-63.87\left(6 \mathrm{~F},-\mathrm{CF}_{3}\right)$.

AF-Ar-PC OH 3: 6FBPA (8.06 g, $24 \mathrm{mmol})$, pyridine $(4.02 \mathrm{~mL})$, triphosgene (2.48 g, $8.33 \mathrm{mmol})$. AF-Ar-PC OH 3 was obtained as a white powder $(5.50 \mathrm{~g}, 55 \%)$. GPC: $M_{\mathrm{n}}=7900 \mathrm{~g} \mathrm{~mol}^{-1} ; M_{\mathrm{w}} / M_{\mathrm{n}}=$ 1.34. IR (KBr, $\left.\mathrm{cm}^{-1}\right): \gamma(\mathrm{C}-\mathrm{F})=1173-1211, \gamma($ aromatic $)=1612$ and 1512, $\gamma(\mathrm{CO}-\mathrm{O}-\mathrm{Ar})=1782 .{ }^{1} \mathrm{H} \mathrm{NMR}\left(500 \mathrm{MHz}, \mathrm{CDCl}_{3}\right)$ : $\delta(\mathrm{ppm}): 7.66-7.64(\mathrm{t}, 4 \mathrm{H}, \mathrm{ArH}), 7.51-7.50(\mathrm{t}, 10 \mathrm{H}, \mathrm{ArH}), 7.40-$ $7.36(\mathrm{~m}, 14 \mathrm{H}, \mathrm{ArH}), 7.27$ (s, 1H, ArH), 6.80 (s, 1H, ArH). ${ }^{13} \mathrm{C} \mathrm{NMR}$ (125 MHz, $\mathrm{CDCl}_{3}$ ), $\delta$ (ppm): 156.22, 151.34, 151.23, 150.47, 138.51, 131.69, 131.28, 128.38, 123.95, 121.36, 120.83, 120.77, 115.16, 64.01. ${ }^{19} \mathrm{~F} \mathrm{NMR} \mathrm{(470} \mathrm{MHz}, \mathrm{CDCl}_{3}, \mathrm{CFCl}_{3}$ ), $\delta$ (ppm): $-63.86\left(6 \mathrm{~F},-\mathrm{CF}_{3}\right)$.

Preparation of epoxy-terminated fluoro-polycarbonates containing aliphatic segments. A round-bottomed flask was charged with PEC-OH and epoxy chloropropane. The reaction mixture was mechanically and vigorously stirred at $55{ }^{\circ} \mathrm{C}$. Then, sodium hydroxide was added every $30 \mathrm{~min}$. The reaction mixture was heated to $60{ }^{\circ} \mathrm{C}$ and stirred for a further $6 \mathrm{~h}$. After the reaction completed, epoxy chloropropane was completely removed under reduced pressure. Then, the residue was dissolved in dichloromethane, followed by removal of sodium chloride by filtration. The products, light yellow viscous liquids, were dried under vacuum for $24 \mathrm{~h}$ and were denoted as AF-Ali-PC EPs.

AF-Ali-PC EP 1:AF-Ali-PC OH 1 (11.59 g, $0.97 \mathrm{mmol}$ ), epoxy chloropropane ( $40 \mathrm{~g}, 0.432 \mathrm{~mol})$, and sodium hydroxide $(0.2 \mathrm{~g}, 5$ mmol). PEC-EP-1 was obtained as a light yellow viscous liquid (11.03 g, 96\%). GPC: $M_{\mathrm{n}}=12001 \mathrm{~g} \mathrm{~mol}^{-1} ; M_{\mathrm{w}} / M_{\mathrm{n}}=1.19$. IR $\left(\mathrm{KBr}, \mathrm{cm}^{-1}\right): \gamma(\mathrm{C}-\mathrm{F})=1170-1251, \gamma($ aromatic $)=1612$ and 1516, $\gamma(\mathrm{CO}-\mathrm{O}-\mathrm{Ar})=1750, \gamma\left(-\mathrm{CH}_{2}\right)=1715$ and $736, \gamma($ epoxy group $)=$ 826. ${ }^{1} \mathrm{H}$ NMR $\left(500 \mathrm{MHz}, \mathrm{CDCl}_{3}\right), \delta(\mathrm{ppm}): 7.34-7.32(\mathrm{t}, 34 \mathrm{H}$, ArH), 6.94-6.92 (t, 34H, ArH), 4.30-4.29 (m, 1H, - $\mathrm{CH}_{2}$ ), 4.00$3.96\left(\mathrm{~m}, 1 \mathrm{H},-\mathrm{CH}_{2}\right), 3.40-3.37(\mathrm{~m}, 1 \mathrm{H},-\mathrm{CH}), 2.95-2.93(\mathrm{~m}, 1 \mathrm{H}$, $\left.-\mathrm{CH}_{2}\right), 2.79-2.78\left(\mathrm{~m}, 1 \mathrm{H},-\mathrm{CH}_{2}\right), 2.41-2.38\left(\mathrm{~m}, 2 \mathrm{H},-\mathrm{CH}_{2}\right), 1.70-$ $1.64\left(\mathrm{~m}, 2 \mathrm{H},-\mathrm{CH}_{2}\right), 1.32-1.29\left(\mathrm{~m}, 6 \mathrm{H},-\mathrm{CH}_{2}\right) .{ }^{13} \mathrm{C}$ NMR (125 $\mathrm{MHz}, \mathrm{CDCl}_{3}$ ), $\delta$ (ppm): 171.93, 158.65, 158.15, 153.64, 131.59, $131.51,126.46,125.96,124.35$, 114.15, 68.80, 63.55, 50.01, 44.64, 34.17, 29.34, 29.17, 29.01, 24.88. ${ }^{19} \mathrm{~F}$ NMR $(470 \mathrm{MHz}$, $\left.\mathrm{CDCl}_{3}, \mathrm{CFCl}_{3}\right), \delta(\mathrm{ppm}):-64.16\left(6 \mathrm{~F},-\mathrm{CF}_{3}\right)$.

AF-Ali-PC EP 2:AF-Ali-PC OH 2 (7.94 g, $0.69 \mathrm{mmol})$, epoxy chloropropane $(27 \mathrm{~g}, 0.276 \mathrm{~mol})$, and sodium hydroxide $(0.138 \mathrm{~g}$, $3.45 \mathrm{mmol})$. PEC-EP-2 was obtained as a light yellow viscous liquid (7.78 g, 96.8\%). GPC: $M_{\mathrm{n}}=11655 \mathrm{~g} \mathrm{~mol}^{-1} ; M_{\mathrm{w}} / M_{\mathrm{n}}=1.29$. IR $\left(\mathrm{KBr}, \mathrm{cm}^{-1}\right): \gamma(\mathrm{C}-\mathrm{F})=1170-1256, \gamma($ aromatic $)=1605$ and $1516, \gamma(\mathrm{CO}-\mathrm{O}-\mathrm{Ar})=1753, \gamma\left(-\mathrm{CH}_{2}\right)=736, \gamma($ epoxy group $)=826$. ${ }^{1} \mathrm{H}$ NMR (500 MHz, $\mathrm{CDCl}_{3}$ ), $\delta$ (ppm): 7.34-7.32 (t, 20H, ArH), 6.94-6.92 (t, 20H, ArH), 4.29-4.28 (m, 1H, $\left.-\mathrm{CH}_{2}\right), 3.99-3.96(\mathrm{~m}$, $\left.1 \mathrm{H},-\mathrm{CH}_{2}\right), 3.39(\mathrm{~s}, 1 \mathrm{H},-\mathrm{CH}), 2.94\left(\mathrm{~s}, 1 \mathrm{H},-\mathrm{CH}_{2}\right), 2.79(\mathrm{~s}, 1 \mathrm{H}$, $\left.-\mathrm{CH}_{2}\right), 2.39\left(\mathrm{~s}, 2 \mathrm{H},-\mathrm{CH}_{2}\right), 1.67-1.66\left(\mathrm{~m}, 2 \mathrm{H},-\mathrm{CH}_{2}\right), 1.29(\mathrm{~s}, 6 \mathrm{H}$, $\left.-\mathrm{CH}_{2}\right) .{ }^{13} \mathrm{C}$ NMR $\left(125 \mathrm{MHz}, \mathrm{CDCl}_{3}\right), \delta$ (ppm): 173.03, 158.65, $158.40,158.15,153.64,131.51,127.78,125.96,124.35,114.15$, $68.49,63.55,50.01,44.63,34.17,29.34,29.17,29.01,24.87 .{ }^{19} \mathrm{~F}$ $\mathrm{NMR}\left(470 \mathrm{MHz}, \mathrm{CDCl}_{3}, \mathrm{CFCl}_{3}\right), \delta(\mathrm{ppm}):-64.16\left(6 \mathrm{~F},-\mathrm{CF}_{3}\right)$.

AF-Ali-PC EP 3:AF-Ali-PC OH 3 (5.04 g, $0.43 \mathrm{mmol})$, epoxy chloropropane (16 g, $0.172 \mathrm{~mol}$ ), and sodium hydroxide (0.086 g, $2.15 \mathrm{mmol}$ ). PEC-EP-3 was obtained as a light yellow viscous liquid (4.88 g, 97.1\%). GPC: $M_{\mathrm{n}}=11683 \mathrm{~g} \mathrm{~mol}^{-1} ; M_{\mathrm{w}} /$ $M_{\mathrm{n}}=1.34$. IR $\left(\mathrm{KBr}, \mathrm{cm}^{-1}\right): \gamma(\mathrm{C}-\mathrm{F})=1170-1246, \gamma($ aromatic $)=$ 1612 and 1516, $\gamma(\mathrm{CO}-\mathrm{O}-\mathrm{Ar})=1743, \gamma\left(-\mathrm{CH}_{2}\right)=743, \gamma($ epoxy group $)=826 .{ }^{1} \mathrm{H}$ NMR $\left(500 \mathrm{MHz}, \mathrm{CDCl}_{3}\right), \delta(\mathrm{ppm}): 7.34-7.32(\mathrm{t}$, $12 \mathrm{H}, \mathrm{ArH}), 6.94-6.92(\mathrm{t}, 12 \mathrm{H}, \mathrm{ArH}), 4.30-4.27\left(\mathrm{~m}, 1 \mathrm{H},-\mathrm{CH}_{2}\right)$, 4.00-3.97 (m, 1H, - $\mathrm{CH}_{2}$ ), 3.40-3.37 (m, 1H, -CH), 2.95-2.93 (m, $\left.1 \mathrm{H},-\mathrm{CH}_{2}\right), 2.80-2.78\left(\mathrm{~m}, 1 \mathrm{H},-\mathrm{CH}_{2}\right), 2.41-2.37\left(\mathrm{~m}, 2 \mathrm{H},-\mathrm{CH}_{2}\right)$, 1.68-1.66 (m, 2H, $\left.-\mathrm{CH}_{2}\right), 1.31-1.29\left(\mathrm{~m}, 6 \mathrm{H},-\mathrm{CH}_{2}\right) .{ }^{13} \mathrm{C}$ NMR (125 
$\mathrm{MHz}, \mathrm{CDCl}_{3}$ ), $\delta$ (ppm): 173.03, 158.64, 158.36, 158.14, 153.60, $131.51,126.22,125.97,124.35,114.14,68.79,63.55,50.01$, 44.65, 34.17, 29.34, 29.17, 29.01, 24.87. ${ }^{19} \mathrm{~F}$ NMR $(470 \mathrm{MHz}$, $\left.\mathrm{CDCl}_{3}, \mathrm{CFCl}_{3}\right), \delta(\mathrm{ppm}):-64.17\left(6 \mathrm{~F},-\mathrm{CF}_{3}\right)$.

Preparation of epoxy-terminated fluoro-polycarbonates containing aromatic segments. The epoxy-terminated fluoropolycarbonates containing aromatic segments were prepared with epoxy chloropropane as described above. The products were denoted as AF-Ar-PC EPs.

AF-Ar-PC EP 1:AF-Ar-PC OH 1 (11.22 g, $1.13 \mathrm{mmol})$, epoxy chloropropane (46 g, $0.503 \mathrm{~mol})$, and sodium hydroxide $(0.23 \mathrm{~g}$, $5.8 \mathrm{mmol}$ ). AF-Ar-PC EP 1 was obtained as a light yellow viscous liquid (10.76 g, 97\%). GPC: $M_{\mathrm{n}}=9923 \mathrm{~g} \mathrm{~mol}^{-1} ; M_{\mathrm{w}} / M_{\mathrm{n}}=1.30$. IR $\left(\mathrm{KBr}, \mathrm{cm}^{-1}\right): \gamma(\mathrm{C}-\mathrm{F})=1176-1254, \gamma($ aromatic $)=1609$ and 1516, $\gamma(\mathrm{CO}-\mathrm{O}-\mathrm{Ar})=1754, \gamma($ epoxy group $)=829 .{ }^{1} \mathrm{H}$ NMR $(500$ $\left.\mathrm{MHz} \mathrm{CDCl}_{3}\right): \delta(\mathrm{ppm}): 7.50-7.49(\mathrm{t}, 3 \mathrm{H}, \mathrm{ArH}), 7.34-7.32(\mathrm{t}, 20 \mathrm{H}$, ArH), 7.01-6.99 (t, 3H, ArH), 6.93-6.92 (t, 20H, ArH), 4.26-4.23 $\left(\mathrm{m}, 2 \mathrm{H},-\mathrm{CH}_{2}\right), 3.94-3.90\left(\mathrm{~m}, 2 \mathrm{H},-\mathrm{CH}_{2}\right), 3.28-3.24(\mathrm{~m}, 2 \mathrm{H},-\mathrm{CH})$, 2.92-2.90 (m, 2H, $\left.-\mathrm{CH}_{2}\right), 2.71-2.70\left(\mathrm{~m}, 2 \mathrm{H},-\mathrm{CH}_{2}\right) .{ }^{13} \mathrm{C}$ NMR $(125$ $\mathrm{MHz}, \mathrm{CDCl}_{3}$ ), $\delta$ (ppm): 158.64, 158.15, 157.68, 153.64, 133.84, 131.58, 131.51, 127.79, 126.39, 125.95, 124.35, 114.94, 114.15, 68.78, 63.64, 50.03, 44.66. ${ }^{19} \mathrm{~F} \mathrm{NMR} \mathrm{(470} \mathrm{MHz}, \mathrm{CDCl}_{3}, \mathrm{CFCl}_{3}$ ), $\delta(\mathrm{ppm}):-64.15\left(6 \mathrm{~F},-\mathrm{CF}_{3}\right)$.

AF-Ar-PC EP 2:AF-Ar-PC OH 2 (8.33 g, $1.03 \mathrm{mmol}$ ), epoxy chloropropane (43 g, $0.459 \mathrm{~mol})$, and sodium hydroxide $(0.21 \mathrm{~g}$, $5.31 \mathrm{mmol}$ ). AF-Ar-PC EP 2 was obtained as a light yellow viscous liquid (7.98 g, 97.2\%). GPC: $M_{\mathrm{n}}=8101 \mathrm{~g} \mathrm{~mol}^{-1} ; M_{\mathrm{w}} / M_{\mathrm{n}}=1.28$. IR $\left(\mathrm{KBr}, \mathrm{cm}^{-1}\right): \gamma(\mathrm{C}-\mathrm{F})=1176-1254, \gamma($ aromatic $)=1612$ and 1516, $\gamma(\mathrm{CO}-\mathrm{O}-\mathrm{Ar})=1759, \gamma($ epoxy group $)=829 .{ }^{1} \mathrm{H}$ NMR $(500$ $\left.\mathrm{MHz} \mathrm{CDCl}_{3}\right): \delta(\mathrm{ppm}): 7.50-7.49(\mathrm{t}, 9 \mathrm{H}, \mathrm{ArH}), 7.34-7.33(\mathrm{t}, 43 \mathrm{H}$, ArH), 7.01-6.99 (t, 9H, ArH), 6.94-6.92 (t, 43H, ArH), 4.25-4.23 $\left(\mathrm{m}, 2 \mathrm{H},-\mathrm{CH}_{2}\right), 3.95-3.92\left(\mathrm{~m}, 2 \mathrm{H},-\mathrm{CH}_{2}\right), 3.27-3.24(\mathrm{~m}, 2 \mathrm{H},-\mathrm{CH})$, 2.93-2.89 (m, 2H, $\left.-\mathrm{CH}_{2}\right), 2.71-2.69\left(\mathrm{~m}, 2 \mathrm{H},-\mathrm{CH}_{2}\right) .{ }^{13} \mathrm{C}$ NMR (125 $\mathrm{MHz}, \mathrm{CDCl}_{3}$ ), $\delta$ (ppm): 158.66, 158.17, 157.70, 153.64, 133.66, 133.83, 131.58, 131.51, 127.78, 125.94, 124.37, 114.96, 114.17, 114.11, 68.79, 63.56, 50.04, 44.62. ${ }^{19} \mathrm{~F}$ NMR (470 $\mathrm{MHz} \mathrm{CDCl}_{3}$, $\left.\mathrm{CFCl}_{3}\right), \delta(\mathrm{ppm}):-64.16\left(6 \mathrm{~F},-\mathrm{CF}_{3}\right)$.

AF-Ar-PC EP 3:AF-Ar-PC OH 3 (5.50 g, $0.696 \mathrm{mmol}$ ), epoxy chloropropane (29 g, $0.310 \mathrm{~mol})$, and sodium hydroxide (0.144 g, $3.59 \mathrm{mmol})$. AF-Ar-PC EP 3 was obtained as a light yellow viscous liquid $(5.23 \mathrm{~g}, 96.5 \%)$. GPC: $M_{\mathrm{n}}=7954 \mathrm{~g} \mathrm{~mol}^{-1}$; $M_{\mathrm{w}} / M_{\mathrm{n}}=1.32$. IR $\left(\mathrm{KBr}, \mathrm{cm}^{-1}\right): \gamma(\mathrm{C}-\mathrm{F})=1176-1254, \gamma($ aromatic $)$ $=1612$ and 1516, $\gamma(\mathrm{CO}-\mathrm{O}-\mathrm{Ar})=1755, \gamma($ epoxy group $)=829 .{ }^{1} \mathrm{H}$ NMR (500 MHz, $\left.\mathrm{CDCl}_{3}\right): \delta(\mathrm{ppm}): 7.50-7.49$ (t, 11H, ArH), 7.34$7.32(\mathrm{t}, 27 \mathrm{H}, \mathrm{ArH}), 7.01-6.99(\mathrm{t}, 11 \mathrm{H}, \operatorname{ArH}), 6.94-6.92(\mathrm{t}, 27 \mathrm{H}$, ArH), 4.27-4.25 (m, 2H, - $\mathrm{CH}_{2}$ ), 3.94-3.91 (m, 2H, $-\mathrm{CH}_{2}$ ), 3.28$3.23(\mathrm{~m}, 2 \mathrm{H},-\mathrm{CH}), 2.95-2.94\left(\mathrm{~m}, 2 \mathrm{H},-\mathrm{CH}_{2}\right), 2.72-2.69(\mathrm{~m}, 2 \mathrm{H}$, $\left.-\mathrm{CH}_{2}\right) .{ }^{13} \mathrm{C}$ NMR (125 MHz, $\mathrm{CDCl}_{3}$ ), $\delta$ (ppm): 158.64, 158.14, 157.69, 153.63, 133.89, 131.60, 131.51, 127.80, 125.98, 125.48, 124.34, 114.95, 114.15, 68.79, 63.75, 50.01, 44.74. ${ }^{19} \mathrm{~F}$ NMR (470 $\left.\mathrm{MHz}, \mathrm{CDCl}_{3}, \mathrm{CFCl}_{3}\right), \delta(\mathrm{ppm}):-64.16\left(6 \mathrm{~F},-\mathrm{CF}_{3}\right)$.

\section{Preparation of the polymer films and their exposure to UV light}

To determine the optical properties and fabricate the optical waveguide, photoresist solutions were prepared by dissolving synthesized AF-Ali-PC EPs or AF-Ar-PC EPs and initiator in cyclopentanone to form 30 to $60 \mathrm{wt} \%$ solutions (FPRs (Ali-PR 13 and Ar-PR 1-3)). FPRs were filtered using a $0.2 \mu \mathrm{m}$ PTFE membrane to remove large particulates and spin-coated onto $\mathrm{Si}$ / $\mathrm{SiO}_{2}$ wafer substrates (500 r/s $9 \mathrm{~s}$ and $1000 \mathrm{r} / \mathrm{s} 30 \mathrm{~s}$ ). The spincoated films were dried on a hot plate for $30 \mathrm{~min}\left(60^{\circ} \mathrm{C}\right)$ and then in a vacuum oven for $12 \mathrm{~h}$ to remove the residual solvent. Then, the films were irradiated with UV light for $6 \mathrm{~min}$ and postbaked at $120{ }^{\circ} \mathrm{C}$ for $30 \mathrm{~min}$ to afford highly transparent films.

\section{Fabrication of the straight waveguide}

To study the applications of Ali-PRs and Ar-PRs in optical waveguides, a straight waveguide was fabricated according to a previously reported method. ${ }^{\mathbf{1 4}}$ To determine the propagation loss of the FPRs, a straight waveguide structure was fabricated on a $\mathrm{Si} / \mathrm{SiO}_{2}$ substrate via direct $\mathrm{UV}$-writing, as shown in Fig. 1(a). After spinning the core solution (FPRs) onto a $\mathrm{Si} / \mathrm{SiO}_{2}$ wafer at $1000 \mathrm{r} / \mathrm{s}$ for $30 \mathrm{~s}$, the coated layers (3-4 $\mu \mathrm{m}$ in thickness) were dried at $110{ }^{\circ} \mathrm{C}$ for $90 \mathrm{~min}$ to remove the residual solvent. Then, the films were exposed to UV light for $6 \mathrm{~min}$ through a contact chromium mask and post-baked at $120^{\circ} \mathrm{C}$ for $30 \mathrm{~min}$. After this, the non-cross-linked FPRs were removed by development in propylene glycol methyl ether acetate (PGMEA) for $5 \mathrm{~s}$ at room temperature, followed by rinsing the wafer in isopropanol. For the upper cladding layer, PMMA was selected. PMMA was spin-coated onto the core layer and baked at $125{ }^{\circ} \mathrm{C}$ for $1 \mathrm{~h}$ to form the cladding film.

\section{Fabrication of the MZI thermo-optic waveguide switch arrays}

The MZI thermo-optic waveguide switch array device was fabricated, as described in Fig. 1(b). First, the gold stripes were patterned by photolithography and wet etching. Second, as described in the "fabrication of the straight waveguide" section in this article, the FPRs were spin-coated onto the gold electrode to form the core layer. Then, a PMMA thin film was formed as the upper cladding layer and was then baked at $125^{\circ} \mathrm{C}$ for $1 \mathrm{~h}$.

\section{Results and discussion}

\section{Synthesis of the polymers}

Epoxy-terminated fluoro-polycarbonates containing aliphatic segments (AF-Ali-PC EPs) and epoxy-terminated fluoropolycarbonates containing aromatic segments (AF-Ar-PC EPs) were synthesized according to Scheme 1 and 2, respectively. The purpose of the functionalization was to incorporate functional groups, such as aliphatic segments, and aromatic segments,

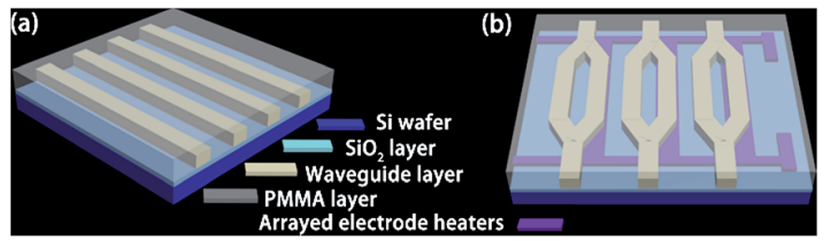

Fig. 1 Schematic of waveguide devices: (a) channel waveguide and (b) MZI thermo-optic waveguide switch arrays. 

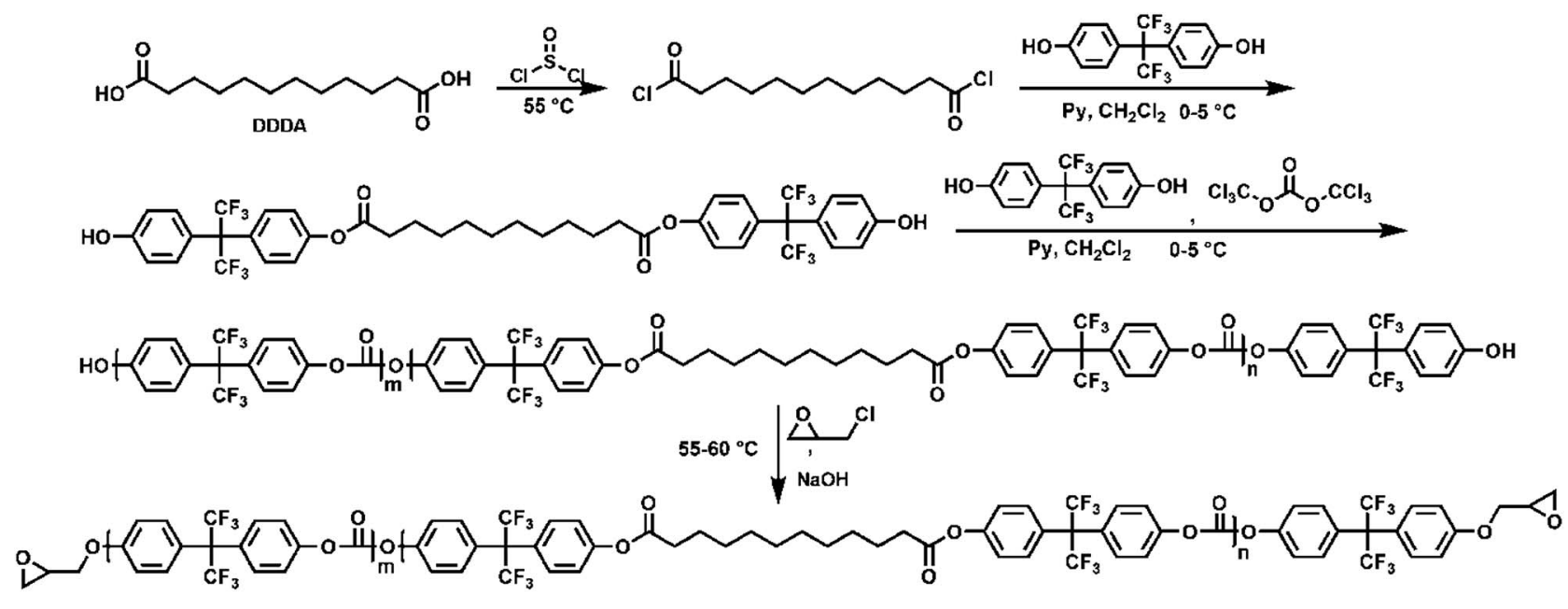

$n(D D D A): n(6 F B P A)=1: 6,1: 10,1: 16$

Scheme 1 The preparation of epoxy-terminated fluoro-polycarbonates containing aliphatic segments (AF-Ali-PC EPs).

into fluoro-polycarbonates chain bearing epoxy units as crosslinking sites.

To acquire AF-Ali-PC EPs, we first synthesized hydroxylterminated fluoro-polycarbonates containing aliphatic segments (AF-Ali-PC OHs). For the purpose of obtaining AF-AliPC OHs, we controlled the molar feed ratio of (6FBPA + DDDA) : BTC at $18: 5$. The structure of AF-Ali-PC OHs was determined by ${ }^{1} \mathrm{H}$ NMR, ${ }^{13} \mathrm{C}$, and DEPT 135 NMR spectroscopy. In the ${ }^{1} \mathrm{H}$ NMR spectra (Fig. S1 $\dagger$ ), the signals of dodecyl in AFAli-PC OHs were observed at 2.62-2.59, 1.82-1.75, and 1.44$1.36 \mathrm{ppm}$. In the ${ }^{13} \mathrm{C}$ and DEPT $135 \mathrm{NMR}$ spectra (Fig. S2 $\dagger$ ), the characteristic peaks of dodecyl in AF-Ali-PC OHs were observed at $34.37,29.39,29.24,29.08$, and $24.86 \mathrm{ppm}$; the $-\mathrm{CF}_{3}$ peaks were observed at $127.37,125.10,122.80$, and $120.81 \mathrm{ppm}$; and the $-\mathrm{C}=\mathrm{O}$ peak was observed at $151.2 \mathrm{ppm}$. The compositions of the copolymers (AF-Ali-PC OHs), calculated from their ${ }^{1} \mathrm{H}$ NMR spectra, were 6 FBPA/DDDA $=12: 1,8: 1$, and $6: 1$ according to the feed ratios. In the next step, we synthesized AFAli-PC EPs with the AF-Ali-PC OHs and epoxy chloropropane. In the ${ }^{1} \mathrm{H}$ NMR spectra (Fig. S1 $\dagger$ ), the signals of the epoxy group in AF-Ali-PC EPs were observed at 4.30-4.27, 3.97-4.00, 3.37-3.40, 2.93-2.95, and 2.78-2.80 ppm; in the ${ }^{13} \mathrm{C}$ and DEPT $135 \mathrm{NMR}$ spectra (Fig. S2 $\dagger$ ), the characteristic peaks of the epoxy group were observed at $68.79,50.01$, and $44.65 \mathrm{ppm}$. These results suggest that the DDDA content of the fluoro-polycarbonates containing aliphatic segments could be readily controlled by adjusting the (6FBPA + DDDA) : BTC feed ratios.

Epoxy-terminated fluoro-polycarbonates containing aromatic segments (AF-Ar-PC EPs) were synthesized according to Scheme 2. The intermediate hydroxyl-terminated fluoropolycarbonates containing aromatic segments (AF-Ar-PC OHs) were synthesized by controlling the molar feed ratio of DOD : BTC at $18: 5$; the structures were determined by ${ }^{1} \mathrm{H}$ NMR, ${ }^{13} \mathrm{C}$, and DEPT 135 NMR spectroscopy. In the ${ }^{1} \mathrm{H}$ NMR spectra (Fig. S7 $\dagger$ ), the signals of diphenyl in AF-Ar-PC OHs were observed at 7.66-7.64 and 7.40-7.36 ppm. In the ${ }^{13} \mathrm{C}$ and DEPT 135 NMR spectra (Fig. S8 $\dagger$ ), the characteristic peaks of diphenyl in AF-Ar-PC OHs were observed at 121.26 and $131.27 \mathrm{ppm}$; the $-\mathrm{CF}_{3}$ peaks were observed at $127.36,125.09,122.81$, and $120.80 \mathrm{ppm}$; and the $-\mathrm{C}=\mathrm{O}$ peak was observed at $151.22 \mathrm{ppm}$. The compositions of the copolymers (AF-Ar-PC OHs), calculated from their ${ }^{1} \mathrm{H}$ NMR spectra, were 6FBPA/DOD = $6: 1,4.33: 1$, and 2.75: 1 according to the feed ratios. The end products AFAli-PC EPs were synthesized using AF-Ali-PC OHs and epoxy chloropropane. In the ${ }^{1} \mathrm{H}$ NMR spectra (Fig. S7 $\dagger$ ), the signals of the epoxy groups in AF-Ar-PC EPs were observed at 4.26-4.23,

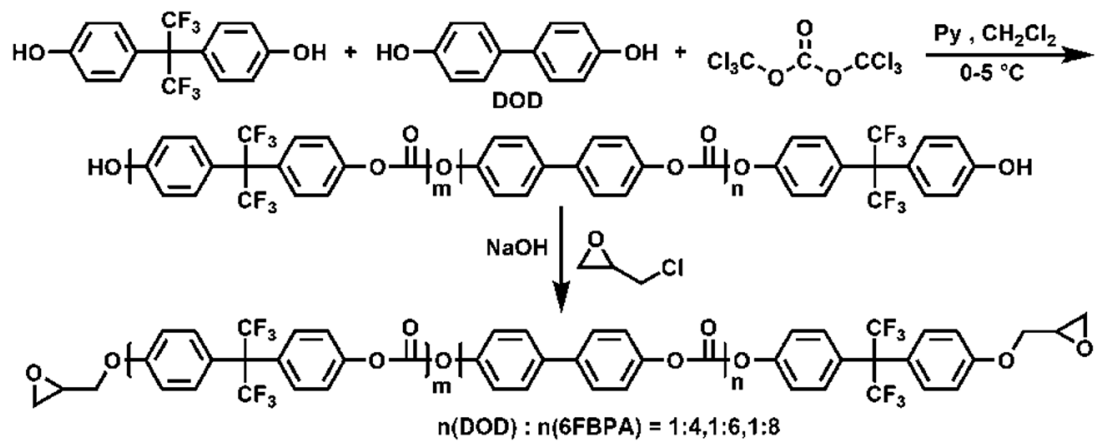

Scheme 2 The preparation of epoxy-terminated fluoro-polycarbonates containing aromatic segments (AF-Ar-PC EPs). 
3.94-3.90, 3.28-3.24, 2.92-2.90, and 2.718-2.70 ppm; in the ${ }^{13} \mathrm{C}$ and DEPT 135 NMR spectra (Fig. S8 $\dagger$ ), the characteristic peaks of the epoxy group were observed at 68.79, 50.04, and $44.62 \mathrm{ppm}$. These results suggest that the DOD content of the fluoro-polycarbonates containing aromatic segments could be readily controlled by adjusting the (6FBPA + DOD) : BTC feed ratio.

\section{The properties of the cross-linking films}

Refractive index. As is known, the theoretical basis of guidedwave optics depends on the phenomenon of total internal reflection (TIR), which can confine light in the optical waveguide. Based on the device design and geometry of the waveguide, the refractive index of the core material that is being used to fabricate the waveguide must be greater than that of the cladding polymer. The controllability of the refractive-index contrast is an important feature of the polymers, which can enable high-density compact waveguiding structures with small radii of curvature. Moreover, a compact structure is critical for achieving large-scale photonic integration. ${ }^{7 f}$ As shown in Fig. 2, we synthesized six types of fluoro-polycarbonates; the refractive indices varied from 1.51 to 1.495 (at $1550 \mathrm{~nm}$ ) for AF-Ali-PC EPs as the content of DDDA was varied from 8 to $14 \mathrm{~mol} \%$, and the refractive indices varied from 1.60 to 1.54 for AF-Ar-PC EPs as the content of DOD was varied from 27 to $14 \mathrm{~mol} \%$. The refractive indices of the polymer films and the DDDA content in AF-Ali-PC EPs or the DOD content in AF-Ar-PC EPs showed linear relationships. For the AF-Ali-PC EPs, the incorporation of flexible segments decreased the refractive indices of similar polymers, whereas for the AF-Ar-PC EPs, the rigid segments increased the refractive indices of similar polymers. ${ }^{15} \mathrm{We}$ could obtain any refractive index between 1.60 and 1.495 by mixing the six types of fluoro-polycarbonates in appropriate proportions.

UV-near infrared spectra. Fig. 3(A) shows the near infrared spectra of the FPR films, and it can be seen that there is almost no absorption between 500 and $2000 \mathrm{~nm}$ compared to that of commercial photoresist SU-8. This result was due to the existence of $\mathrm{C}-\mathrm{F}$ bonds in the FPRs. As is known, $\mathrm{C}-\mathrm{H}, \mathrm{O}-\mathrm{H}$, and $\mathrm{N}-\mathrm{H}$ bonds can cause absorption in the near-IR region. When $\mathrm{H}$

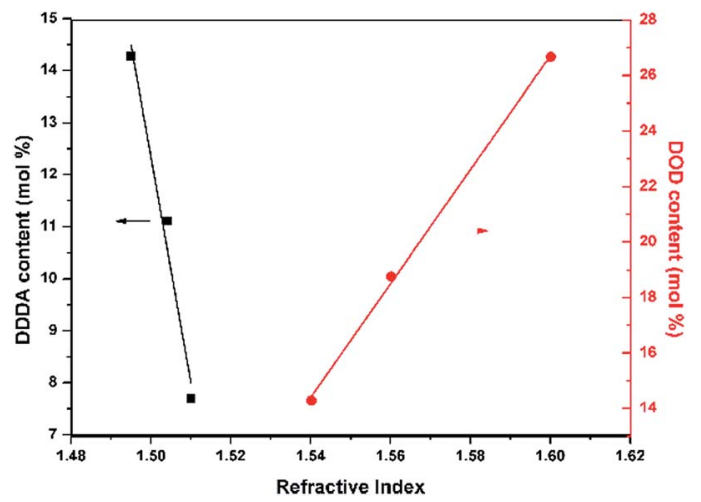

Fig. 2 Relationships between the refractive indices of the polymer films and the content of DDDA/DOD. atoms are replaced with $\mathrm{F}$ atoms, the absorption in the near-IR region can be significantly reduced. For Ali-PR 1-3, the absorbance values at $1550 \mathrm{~nm}$ increased with the increasing DDDA content in AF-Ali-PC EP 1-3 (Fig. 3(A)(1)-(3) and (B)). Moreover, for Ar-PR 1-3, the absorbance values at $1550 \mathrm{~nm}$ increased with the increasing content of DOD in AF-Ar-PC EP 1-3 (Fig. 3(A)(4)(6) and (B)). This result was mainly due to the different contents of fluorine $(31.12 \mathrm{wt} \%, 30.03 \mathrm{wt} \%$, and $28.16 \mathrm{wt} \%$ for AF-Ar-PC EP 1-3, respectively) since the absorbance values decreased with the decreasing content of F, which corresponds with the abovementioned conclusion. Due to their lower absorptions at telecommunication wavelengths (1310 $\mathrm{nm}$ and $1550 \mathrm{~nm}$ ), FPRs are more suitable for application in optical waveguide devices.

Surface morphology. The surface morphologies of the FPR films were determined by atomic force microscopy (AFM). As presented in Fig. 4(A) and (B), the surface morphologies of the non-cross-linked (A) and cross-linked (B) films on the surface of Ali-PR 1 were very homogeneous, and we could not observe apparent defects, and the surface roughnesses were only $0.335 \mathrm{~nm}$ and $0.371 \mathrm{~nm}$, respectively. This result indicates that the quality of the films, which were formed from Ali-PRs, was good. In addition, it can be suggested that the waveguide devices fabricated with Ali-PRs will have a smaller scattered light loss. Fig. 4(C) and (D) show the surface morphologies of the non-crosslinked (C) and cross-linked (D) films on the surface of Ar-PR 1, and the surface roughnesses were $0.293 \mathrm{~nm}$ and $2.593 \mathrm{~nm}$, respectively. Owing to the presence of biphenyl structures in the polycarbonate chains, the typical droplet-like morphology with an average size of $1 \mu \mathrm{m}$ was formed during the process of preparation of the polymer films and UV exposure. ${ }^{\mathbf{1 6}}$

Solubility and thermal properties. The introduction of an aliphatic segment (AF-Ali-PC EPs) would increase the mobility and thus the flexibility of the polycarbonate chains, ${ }^{17}$ which could effectively improve their solubility compared to that of polycarbonates containing aromatic segments (AF-Ar-PC EPs). However, the cross-linked films of both polymers were all insoluble due to the formation of crosslinking network structures. We soaked the cross-linked polymer films in chloroform for a week. Then, we obtained the weights of the films before and after soaking. The results are given in Table 1. After soaking, there was less than $0.05 \%$ weight loss, which indicates that the cross-linked polymers have good resistance to organic solvents.

The thermal properties of the FPRs were evaluated by TGA and DSC measurements (Fig. S5, S6, S11 and S12†). The thermal decomposition behaviors and glass transition temperatures were measured under nitrogen, and the relevant results are summarized in Table 1 . Weight losses of less than $5 \%$ were observed up to $260{ }^{\circ} \mathrm{C}$ before cross-linking and up to $305{ }^{\circ} \mathrm{C}$ after cross-linking for Ali-PRs, whereas those for Ar-PRs were observed up to $263{ }^{\circ} \mathrm{C}$ before cross-linking and up to $299{ }^{\circ} \mathrm{C}$ after cross-linking. The $T_{\mathrm{g}} \mathrm{s}$ of the non-cross-linked FPRs were observed in the range of $7.3-22.8{ }^{\circ} \mathrm{C}$. Since the flexibility of the polymer chains decreased through UV light curing, no $T_{\mathrm{g}}$ was observed after cross-linking. These results indicate that all the FPRs have excellent thermal stabilities, which are sufficient for practical applications in optical waveguides. 

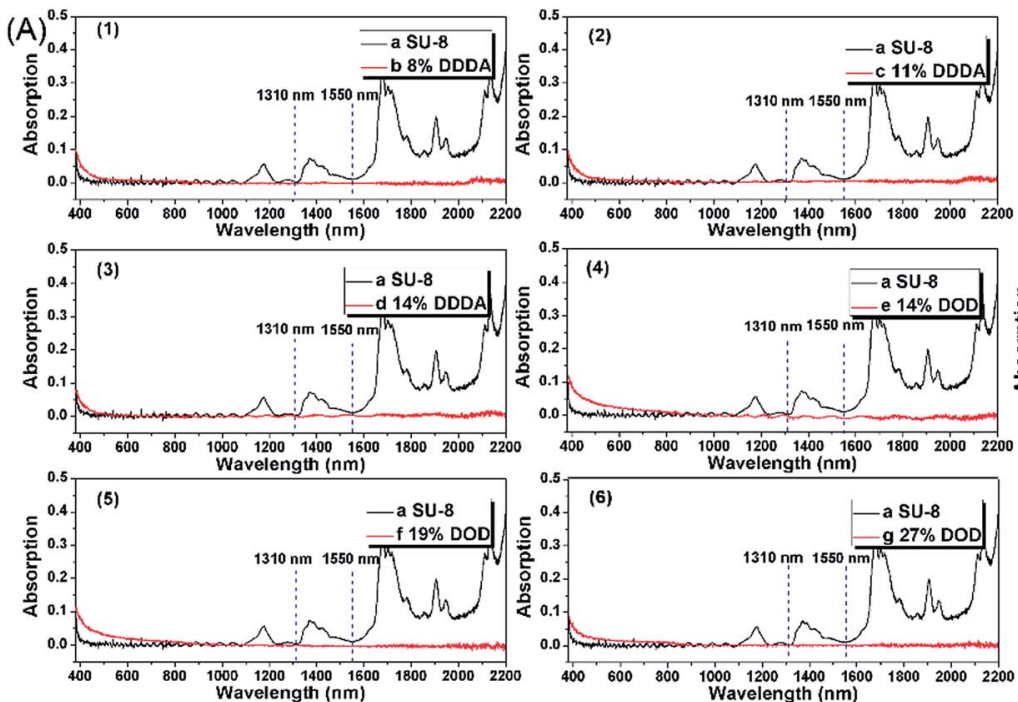

(B)

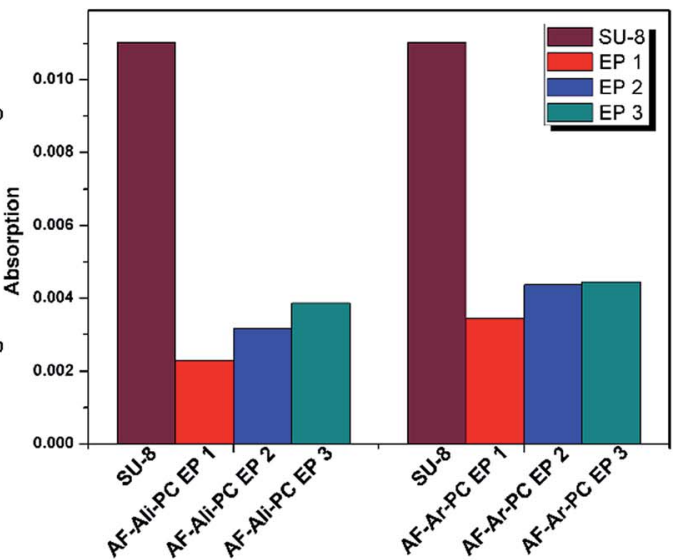

Fig. 3 (A) Near-infrared absorption spectra of Ali-PR 1-3 (b-d), Ar-PR 1-3 (e-g), and SU-8 (a). (B) The absorbance values at $1550 \mathrm{~nm}$ of AF-AliPC EP 1-3 and AF-Ar-PC EP 1-3.

\section{Straight optical waveguide and propagation loss} measurements

Typical near-field patterns of the straight waveguide at the wavelength of $1550 \mathrm{~nm}$ are shown in Fig. 5(A) and (B) for Ali-PR 1 and Ar-PR 1, respectively. The propagation losses at $1550 \mathrm{~nm}$, which were obtained from the slope of the optical insertion loss versus the waveguide length curves by the cut-back method, were $0.21 \mathrm{~dB} \mathrm{~cm}^{-1}$ and 0.19 $\mathrm{dB} \mathrm{cm}{ }^{-1}$.

\section{MZI thermo-optic waveguide switch arrays and thermo-optic} characterization

Fig. 6(A) and (B) show the results of the thermo-optic switching response. The rise and fall times of the thermo-optic switch
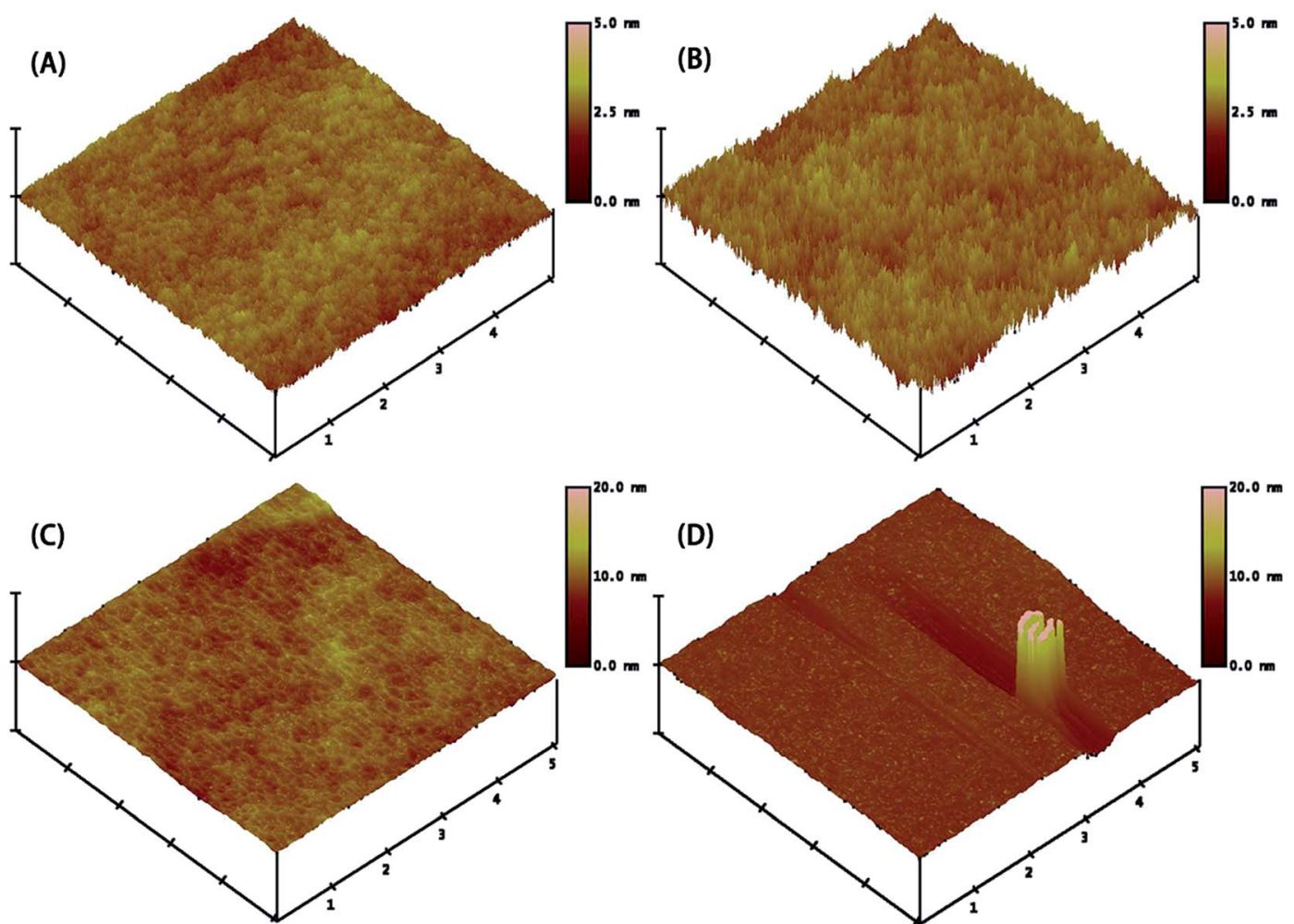

Fig. 4 AFM images of the surfaces of Ali-PR 1 (A) before cross-linking and (B) after cross-linking ( $5 \times 5 \mu \mathrm{m})$ and Ar-PR 1 (C) before cross-linking and $(D)$ after cross-linking $(5 \times 5 \mu \mathrm{m})$. 
Table 1 Characterization and properties of the fluorinated polycarbonates

\begin{tabular}{|c|c|c|c|c|c|c|}
\hline Polymer & $\begin{array}{l}\text { Molar feed ratio } \\
\text { 6FBPA to DDDA/DOD }\end{array}$ & $\begin{array}{l}\text { Actual molar ratio } \\
\text { 6FBPA to DDDA/DOD }\end{array}$ & $T_{\mathrm{g}}^{a}\left({ }^{\circ} \mathrm{C}\right)$ & $T_{\mathrm{d}}^{b}\left({ }^{\circ} \mathrm{C}\right)$ & $T_{\mathrm{d}}^{c}\left({ }^{\circ} \mathrm{C}\right)$ & $\begin{array}{l}\text { Gel fraction }{ }^{d} \\
(\%)\end{array}$ \\
\hline AF-Ali-PC EP 1 & $16: 1$ & $12: 1$ & 22.8 & 268 & 305 & 99.98 \\
\hline AF-Ali-PC EP 2 & $10: 1$ & $8: 1$ & 22.0 & 260 & 313 & 99.96 \\
\hline AF-Ali-PC EP 3 & $6: 1$ & $6: 1$ & 21.8 & 272 & 306 & 99.97 \\
\hline AF-Ar-PC EP 1 & $8: 1$ & $6: 1$ & 7.3 & 274 & 299 & 99.95 \\
\hline AF-Ar-PC EP 2 & $6: 1$ & $4.33: 1$ & 8.4 & 277 & 303 & 99.97 \\
\hline AF-Ar-PC EP 3 & $4: 1$ & $2.75: 1$ & 13.5 & 263 & 308 & 99.96 \\
\hline
\end{tabular}

${ }^{a}$ Determined at the heating rate of $10{ }^{\circ} \mathrm{C} \mathrm{min}^{-1}$ under nitrogen by DSC. ${ }^{b}$ Defined as the weight loss of the non-cross-linked polymers at 5 wt $\%$, which was determined at the heating rate of $10^{\circ} \mathrm{C} \mathrm{min}{ }^{-1}$ under nitrogen by TGA. ${ }^{c}$ Defined as the weight loss of the cross-linked polymers at $5 \mathrm{wt} \%$, which was determined at the heating rate of $10^{\circ} \mathrm{C} \mathrm{min}{ }^{-1}$ under nitrogen by TGA. ${ }^{d}$ Calculated from the weight ratio of the cross-linked polymers from chloroform: initial weight.
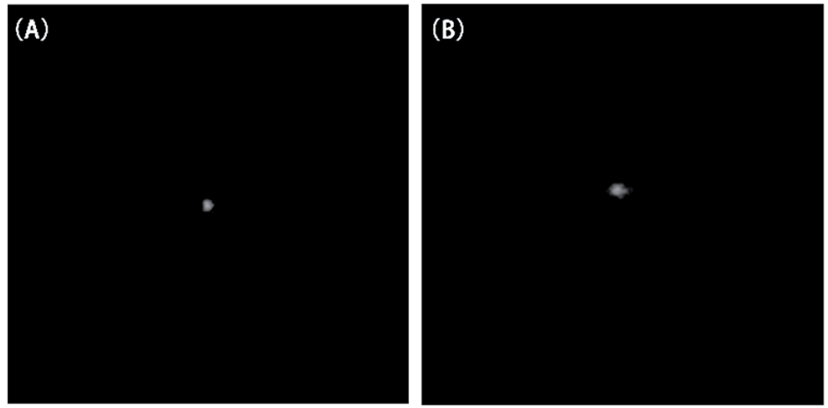

Fig. 5 Near-field patterns of the straight waveguide at $1550 \mathrm{~nm}$ (A) for Ali-PR 1 and (B) for Ar-PR 1.

upon application of a $200 \mathrm{~Hz}$ square-wave voltage were 1.822 $\mathrm{ms}$ and $1.364 \mathrm{~ms}$, respectively, for Ali-PRs and $2.994 \mathrm{~ms}$ and $2.301 \mathrm{~ms}$, respectively, for Ar-PRs at the square-wave voltage of $110 \mathrm{~Hz}$. The insertion loss and extinction ratio were measured to be $9.2 \mathrm{~dB}$ and $12.0 \mathrm{~dB}$ for the aliphatic segment-containing photoresists and $8.9 \mathrm{~dB}$ and $12.3 \mathrm{~dB}$ for the aromatic segmentcontaining waveguides, respectively. The applied electric powers were $15.0 \mathrm{~mW}$ and $20.0 \mathrm{~mW}$, which were also the switching powers. Combined with our previous work ${ }^{9}$ (AF-Z-PC EPs: insertion loss $9.0 \mathrm{~dB}$, rise and fall times $1.546 \mathrm{~ms}$ and $1.226 \mathrm{~ms}(200 \mathrm{~Hz})$, switching power $15.5 \mathrm{~mW}$, and extinction ratio $13.0 \mathrm{~dB}$ ), we can predict that the fluoro-polycarbonates containing aliphatic segments are more suitable for thermooptic waveguide switches than those containing aromatic chains.

\section{Conclusions}

In conclusion, to investigate the effects of the flexibility of the polymer chain on the characteristics of thermo-optic waveguide devices, a series of fluoro-polycarbonates were designed and successfully prepared in this study. As reported in our previous work, photocuring of solutions (Ali-PRs and Ar-PRs) containing AF-Ali-PC EPs or AF-Ar-PC EPs, a photoinitiator, and cyclopentanone yielded optical films with low surface energies and roughnesses, high thermal stabilities, and tunable refractive indices. Straight optical waveguide and MZI thermo-optic waveguide switch arrays were fabricated using Ali-PR 1 and Ar-PR 1. The propagation losses of the channel waveguide measured by the cutback method were $0.21 \mathrm{~dB} \mathrm{~cm}^{-1}$ and 0.19 $\mathrm{dB} \mathrm{cm} \mathrm{cm}^{-1}$ at $1550 \mathrm{~nm}$. For Ali-PRs, the rise and fall times of the thermo-optic switch upon application of a $200 \mathrm{~Hz}$ square-wave voltage were $1.822 \mathrm{~ms}$ and $1.364 \mathrm{~ms}$, respectively. The insertion loss and extinction ratio were measured to be $9.2 \mathrm{~dB}$ and 12.0 dB, respectively. The applied electric power was $15.0 \mathrm{~mW}$. For Ar-PRs, the rise and fall times of the thermo-optic switch
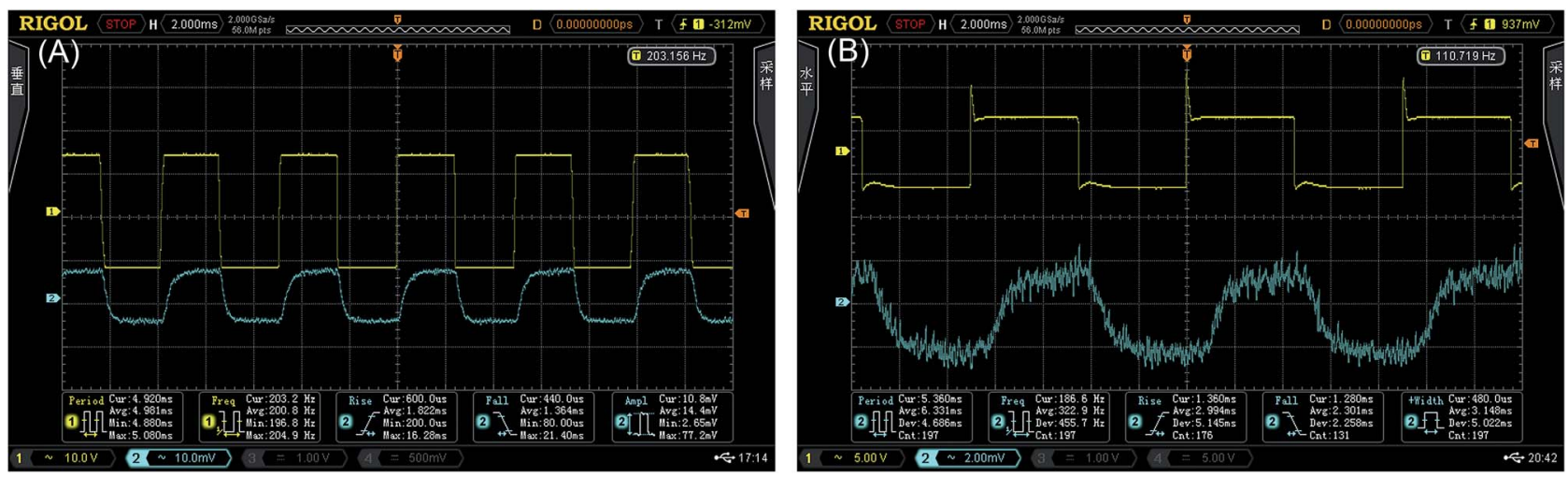

Fig. 6 Thermo-optic switch responses obtained by applying a square-wave voltage at the frequencies of $200 \mathrm{~Hz}$ for Ali-PR 1 (A) and at the 110 $\mathrm{Hz}$ for Ar-PR 1 (B). 
upon application of a $110 \mathrm{~Hz}$ square-wave voltage were $2.994 \mathrm{~ms}$ and $2.301 \mathrm{~ms}$, respectively. The insertion loss and extinction ratio were measured to be $8.9 \mathrm{~dB}$ and $12.3 \mathrm{~dB}$, respectively. The applied electric power was $20.0 \mathrm{~mW}$. Compared AF-Ali-PC EPs with AF-Ar-PC EPs, fluoro-polycarbonates containing aliphatic segments were more suitable for use in optical waveguide devices than those containing aromatic segments; thus, they may be more attractive for improving stabilities and realizing cross-connectors for applications in large-scale PICs such as OXC and OADM systems.

\section{Acknowledgements}

The authors are grateful for the support received from the National Natural Science Foundation of China (Grant No. 21204028, 21221063 and 51173063) and Project 2016159 supported by the Graduate Innovation Fund of Jilin University.

\section{Notes and references}

1 (a) L. Lu, L. Zhou, Z. Li, D. Li, S. Zhao, X. Li and J. Chen, IEEE Photonics Technol. Lett., 2015, 27, 2457; (b) R. G. Beausoleil, M. McLaren and N. P. Jouppi, IEEE J. Sel. Top. Quantum Electron., 2013, 19, 3700109; (c) A. Shacham, K. Bergman and L. P. Carloni, IEEE Trans. Comput., 2008, 57, 1246.

2 F. Qiu, Q. Wang, D. Yang, G. Cao, Y. Guan and L. Zhuang, Eur. Polym. J., 2013, 49, 2247.

3 F. Qiu, J. Liu, G. Cao, Y. Guan, Q. Shen, D. Yang and Q. Guo, Opt. Commun., 2013, 296, 53.

4 (a) B. Lee, C. Lin, X. Wang, R. T. Chen, J. Luo and A. K. Y. Jen, Opt. Lett., 2009, 34, 3277; (b) D. Perron, M. Wu, C. Horvath, D. Bachman and V. Van, Opt. Lett., 2011, 36, 2731; (c) A. M. Al-hetar, A. B. Mohammad, A. S. M. Supa'at, Z. A. Shamsan and I. Yulianti, Opt. Commun., 2011, 284, 1181.

5 C.-Y. Wu, P. Lin, R.-S. Huang, W.-C. Chao and M. M. H. Lee, Appl. Phys. Lett., 2006, 89, 121121.

6 Z. Cao, L. Jin, Y. Liu, Z. Jiang and D. Zhang, J. Appl. Polym. Sci., 2013, 127, 607.

7 (a) Y.-C. Chen, H.-L. Chang, R.-H. Lee, S. A. Dai, W.-C. Su and R.-J. Jeng, Polym. Adv. Technol., 2009, 20, 493; (b) J.-S. Koo, P. G. R. Smith and R. B. Williams, Chem. Mater., 2002, 14, 5030; (c) M. Jang, C. K. Park and N. Y. Lee, Sens. Actuators, B , 2014, 193, 599; (d) A. Podzorov and G. Gallot, Appl. Opt., 2008, 47, 3254; (e) C.-C. Chang and W.-C. Chen, Chem. Mater., 2002, 14, 4242; $(f)$ H. Ma, A. K.-Y. Jen and L. R. Dalton, Adv. Mater., 2002, 14, 1339; $(g)$ E. Kim, S. Y. Cho, D. M. Yeu and S. Y. Shin, Chem. Mater., 2005, 17,
962; (h) W.-S. Kim, K.-S. Kim, Y.-J. Eo, K. B. Yoon and B.-S. Bae, J. Mater. Chem., 2005, 15, 465; (i) W. H. Wong, K. S. Chan and E. Y. B. Pun, Appl. Phys. Lett., 2005, 87, 011103; (j) C. M. Chen, X. Z. Zhang, H. M. Zhang, D. Zhang, S. K. Mu, A. L. Hou, X. L. Zhang, L. Li and D. M. Zhang, Microw. Opt. Tech. Lett., 2007, 49, 3040.

8 (a) J. T. Bendler, C. A. Edmondson, M. C. Wintersgill, D. A. Boyles, T. S. Filipova and J. J. Fontanella, Eur. Polym. J., 2012, 48, 830; (b) G. Deng, H. Huang, P. Si, H. Xu, J. Liu, S. Bo, X. Liu, Z. Zhen and L. Qiu, Polymer, 2013, 54, 6349.

9 Z. Cai, B. Wang, Y. Zheng, M. Li, Y. Li, C. Chen, D. Zhang, Z. Cui and Z. Shi, J. Mater. Chem. C, 2016, 4, 533.

10 (a) Y. Wan, Y. Zhang, Z. Shi, W. Xu, X. Zhang, L. Zhao and Z. Cui, Polymer, 2012, 53, 967; (b) J. Ding, J. Jiang, C. Blanchetiere and C. L. Callender, Macromolecules, 2008, 41, 758; (c) K. T. Powell, C. Cheng and K. L. Wooley, Macromolecules, 2007, 40, 4509; (d) S. Wong, H. Ma, A. K. Y. Jen, R. Barto and C. W. Frank, Macromolecules, 2004, 37, 5578.

11 (a) M.-C. Oh, K.-J. Kim, W.-S. Chu, J.-W. Kim, J.-K. Seo, Y.-O. Noh and H.-J. Lee, Polymer, 2011, 3, 975; $(b)$ H. Ma, J. Luo, S. H. Kang, S. Wong, J. W. Kang, A. K. Y. Jen, R. Barto and C. W. Frank, Macromol. Rapid Commun., 2004, 25, 1667; (c) S. H. Kang, J. Luo, H. Ma, R. R. Barto, C. W. Frank, L. R. Dalton and A. K.-Y. Jen, Macromolecules, 2003, 36, 4355; (d) M. Faccini, M. Balakrishnan, R. Torosantucci, A. Driessen, D. N. Reinhoudt and W. Verboom, Macromolecules, 2008, 41, 8320.

12 (a) H. Mochizuki, T. Mizokuro, N. Tanigaki, T. Hiraga and I. Ueno, Polym. Adv. Technol., 2005, 16, 67; (b) T. Takahashi, K. Yonetake, K. Koyama and T. Kikuchi, Macromol. Rapid Commun., 2003, 24, 763; (c) X. Chang, T. Ding, H. Tian and T. Wang, J. Appl. Polym. Sci., 2016, 43636.

13 Z. Cai, H. Yu, Y. Zhang, M. Li, X. Niu, Z. Shi, Z. Cui, C. Chen and D. Zhang, Polymer, 2015, 61, 140.

14 Y. Zheng, C. M. Chen, Y. L. Gu, D. M. Zhang, Z. Z. Cai, Z. S. Shi, X. B. Wang, Y. J. Yi, X. Q. Sun, F. Wang and Z. C. Cui, Opt. Mater. Express, 2015, 5, 1934.

15 H. R. Allcock, J. D. Bender and Y. Chang, Chem. Mater., 2003, 15, 473.

16 A. Tercjak, E. Serrano, M. Larranaga and I. Mondragon, J. Appl. Polym. Sci., 2008, 108, 1116.

17 B. A. Sweileh, H. R. Al-Qalawi and H. A. Mohammad, J. Appl. Polym. Sci., 2014, 131, 39904. 